\title{
3D continuum damage approach for simulation of crack initiation and growth in ceramic materials
}

\author{
B. Michel ${ }^{1, a}$, TH. Helfer ${ }^{1, b}$, I. Ramière ${ }^{1, c}$ and C. Esnoul ${ }^{2, d}$ \\ ${ }^{1}$ CEA DEN/DEC/SESC bat 151 CEA de Cadarache 13108 Saint Paul Lez Durance FRANCE \\ ${ }^{2}$ EDF R\&D - Site des Renardières - Dpt MMC 77818 Moret sur Loing - France \\ abruno.michel@cea.fr, ${ }^{b}$ thomas.helfer@cea.fr, ${ }^{c}$ isabelle.ramiere@cea.fr, ${ }^{\mathrm{d}}$ coralie.esnoul@edf.fr
}

Keywords: Crack initiation and growth, numerical simulation, damage model.

\begin{abstract}
This paper focuses on the numerical simulation of crack initiation and growth in ceramic materials. This work is devoted to nuclear fuel modelling under irradiation and more precisely to fuel pellet fragmentation assessment at macroscopic and microscopic scales. Simulation tools are developed in the framework of a cooperative program between the CEA, EDF and AREVA devoted to a unified fuel performance software environment called PLEIADES.

A smeared crack model is proposed to have a continuous description of crack nucleation and growth at macroscopic scale. This unified description is based on crack extension process from the microscopic scale up to the macroscopic scale. In order to deal with unstable crack extension a specific algorithm is proposed to solve the quasi static nonlinear mechanical problem. A 3D application is presented to illustrate performances and robustness of the smeared crack approach to simulate crack extension in nuclear fuel ceramics. In this application with an internal pressure loading a new methodology is proposed in order to avoid convergence problem due to the indetermination of the quasi static formulation of a softening material equilibrium under Neumann boundary condition.
\end{abstract}

\section{Introduction}

Numerical simulation of crack initiation and growth in nuclear fuel ceramics is an important issue to assess mechanical integrity of the first confinement barrier for radio elements. Under irradiation fuel pellet fragmentation has to be considered because it will have an impact on the loading level and can increase the risk of rupture for the fuel element cladding. Since years 2000 this has been studying [1], [2] for Pellet Cladding Interaction (PCI) phenomenon [3] in fuel rods of Pressurized Water Reactors (PWRs) in order to take into account the impact of pellet fragmentation in Stress Corrosion Cracking cladding rupture [4]. Pellet fragmentation at microscopic scale is also an important issue for fuel dispersal risk under Loss Of Coolant Accident (LOCA) transient loading condition [6]. A smeared crack model [1], [2] has been proposed and implemented in the fuel performance software environment PLEIADES [3] developed in the framework of a cooperative program, between EDF, AREVA and the CEA. This approach has been used for many years to simulate crack extension in the 1D, 2D and 3D fuel models of the PWR fuel performance code ALCYONE [5] of the PLEIADES platform.

The objective of this paper is to present an overview of this modelling approach and a set of new results of 3D simulation for complex crack path extension. In a first part, the model and numerical approach will be detailed. Then, 3D simulation results will be discussed for crack extension in fuel pellet under thermal gradient and for microscopic fuel fragmentation under gaseous fission products internal pressure.

\section{Smeared crack model}

The formulation of the smeared crack model is given with equations (1) to (3). The function $g()$ is defined has a cohesive zone model but it is used as a continuous damage approach where the crack strain is derived from the displacement discontinuity with a characteristic length L. The cohesive 
zone model $g()$ is able to represent softening from microscopic crack growth up to a macroscopic rupture where the material lost its mechanical stiffness. Crack closure due to unloading is also possible with the model. If unloading occurred during the softening stage, the unloading path is assumed to be elastic with an affine linear stiffness. It means that local plasticity due microscopic cracks is negligible, which seems reasonable for fragile rupture. After a complete crack closure the material can recover its elastic stiffness under a compressive stress state defined in the fracture basis. In this paper the fracture basis is derived from the stress tensor's eigenvalues at crack initiation. At this stage we didn't introduce constitutive equations to model material softening under a shear strain loading occurring in the fracture basis after crack initiation. In this formulation it is assumed that the stress eigen basis doesn't change during the softening process (radial stress relaxation) and that shear stresses can be neglected.

$$
\begin{aligned}
& \overline{\bar{\sigma}}=\underline{\underline{E}}:\left(\begin{array}{l}
\overline{\dot{\varepsilon}}^{\text {tot }}-\overline{\dot{\varepsilon}^{\text {crack }}} \\
=
\end{array}\right) \\
& ={ }^{\text {crack }}=\sum_{i} \varepsilon_{i}^{\text {crack }} \cdot \vec{w}_{i} \otimes \vec{w}_{i} \\
& \left.=={ }^{\text {crack }}\right)=0 \\
& g\left(\sigma: n_{i}, L \cdot \varepsilon_{i}^{\text {cras }}\right)=0
\end{aligned}
$$

Where $\underline{\underline{E}}$ is the fourth order elastic tensor, $\overline{\dot{\varepsilon}}^{\text {crack }}$ is the crack strain rate tensor, $\vec{w}_{i}$ is the unit vectors used to define an orthonormal crack basis, L is the characteristic length for the regularization method and $g()$ is the cohesive zone model for fragile rupture.

\section{Numerical approach}

As presented in reference [2] the numerical integration of the local smeared crack model is based on an implicit approach implemented in the Cast3M Finite Element code [7] through the MFront material knowledge integrator [8]. A quasi-static approach of the equilibrium under unstable crack extension leads to a reverse displacement solution (snap back) needed to decrease elastic energy restitution rate up to the dissipated energy rate. In order to be able to solve the numerical problem associated to unstable crack extension a new approach has been proposed. This methodology is based on a modified Newton algorithm (MNA) to solve the nonlinear problem associated to the quasi static equilibrium formulation. The main idea of this methodology is based on the fact that the solution reached after unstable crack extension will be consistent again with a static equilibrium if external loading is based on Dirichlet boundary conditions. If we compare to a full dynamic solution it means that the post-instability static solution is consistent with a state where the kinetic energy stored during the damage process is vanished because of material dumping coupling with energy transport by stress waves. In order to be able to find this post-instability static solution a specific algorithm is needed because the MNA can't converge towards this solution if the iterative process is initialized with the elastic solution at crack initiation. This specific algorithm is based on two different aspects, first an adaptative time step algorithm, and secondly a fictive path loading algorithm. Thanks to the time step refinement method the unstable crack extension stage starts from the crack initiation loading. This time step refinement method is based on the convergence of the MNA, in order to detect if the unstable crack extension stage has been reached or not. The time step reduction ratio can be set in order to reach a given precision for the crack initiation criterion at the beginning of the unstable crack extension stage; this precision has to be consistent with the convergence criterion used in the MNA. To find the post instability static solution, intermediate updating of the mechanical state will be validated in the MNA even if these solutions are only consistent with the material behaviour and not with the static equilibrium. The residual equilibrium error associated to these intermediate up-dating can be considered as the dynamic nodal forces needed to reach a dynamic equilibrium. 


\section{Crack initiation and growth at microscopic scale}

In nuclear fuel under irradiation gaseous fission products tend to precipitate in small bubbles and can induce internal stresses in the porous material. To study the risk of fuel pellet microscopic fragmentation we have developed a 3D volume element model of the nuclear fuel [6]. This finite element model, derived from a MOX fuel microstructure, is based on a periodic volume element of the porous material. Bubble sizes statistical distribution is defined with post irradiation micrographic examinations, and bubble spatial localization statistical distribution is derived from a Random Sequential Addition algorithm. Input parameter are given in Table 1, the effective material tenacity is an equivalent value taking into account a reduction of the real material tenacity due to the pressure driven forces on the crack surfaces. In order to avoid an indeterminacy of the static solution under Neumann boundary condition, the pressure loading is replaced by a strain control loading. The latter is defined through a fictive thermal expansion of the bubble gas, itself considered as a linear elastic material with a shear modulus much lower than the bulk modulus. As presented in equation (8) this leads to a pure hydrostatic stress state in the gas, and the stress vector at the gas-solid material interface is equivalent to a pressure loading boundary condition. Gas equivalent properties used in our study are given in Table 1 and the results obtained for both types of loading conditions are given in Figure 1. As shown in the latter, the fictive thermal loading leads to the same stress sate in the solid material and the local error between the two stress fields is lower than $1 \%$.

$$
\begin{aligned}
& \overline{\bar{\sigma}}=2 . \mu . \bar{\varepsilon}+\lambda \cdot \operatorname{tr}(\bar{\varepsilon}) . \overline{\bar{I}} \\
& \mu<<\lambda \Rightarrow \overline{\bar{\sigma}} \approx-p . \overline{\bar{I}}
\end{aligned}
$$

Numerical results for microscopic fuel fragmentation are presented in Figure 2 with an amplification of the displacement field and a suppression of damaged element. The mean pressure in bubbles increases up to $120 \mathrm{MPa}$ when a first unstable crack extension occurred. After the first crack extension the pressure is no more homogeneous in bubbles. Bubbles crossed by or near a crack plane have a reduced pressure due to the softening of damaged material. This leads to a mean pressure drop for each new crack extension has shown in Figure 2. Through these results we can compute the fuel fragment size distribution or the amount of gas released has a function of the material microstructure and the pressure induced by gaseous fission products.

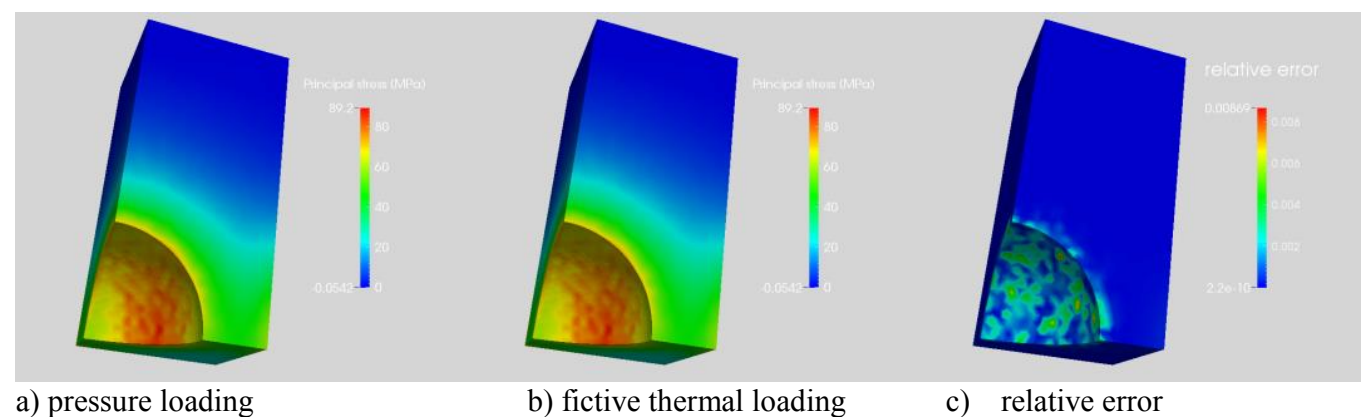

a) pressure loading

b) fictive thermal loading

c) relative error

Figure 1 : Maximal principal stress induced by internal pressure.

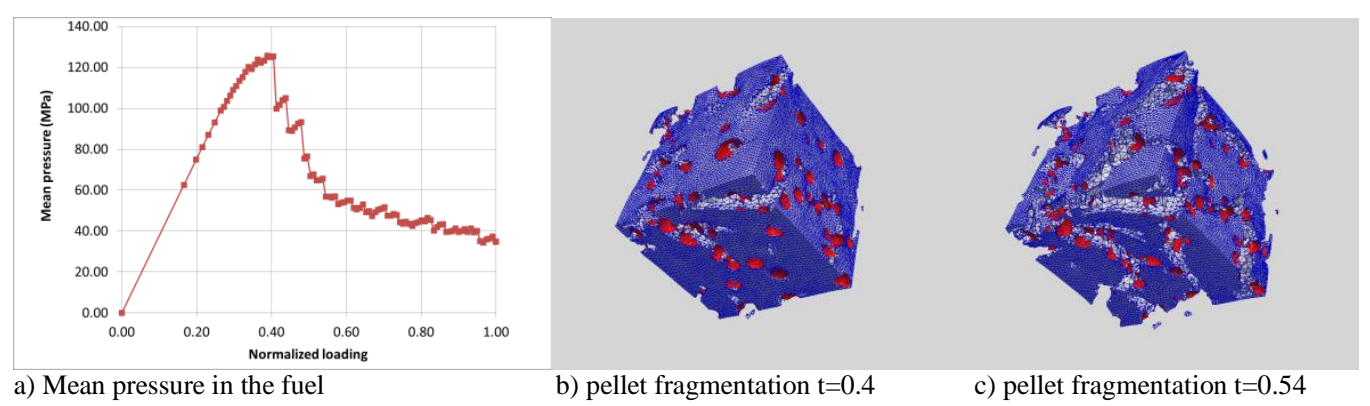

Figure 2 : Numerical results for fuel fragmentation at microscopic scale. 


\begin{tabular}{|c|c|c|c|c|c|c|c|}
\hline \multicolumn{2}{|c|}{ Fuel Volume element } & \multicolumn{3}{c|}{ Solid material properties } & \multicolumn{2}{c|}{ Gas equivalent properties } \\
\hline Size & $\begin{array}{c}\text { Bubble volumique } \\
\text { fraction ratio }\end{array}$ & Young modulus & $\begin{array}{c}\text { Poisson } \\
\text { ratio }\end{array}$ & Rupture stress & $\begin{array}{c}\text { Effective material } \\
\text { tenacity }\end{array}$ & $\lambda$ & $\mu$ \\
\hline $9 \mu \mathrm{m}$ & $8 \%$ & $200000 \mathrm{MPa}$ & 0.3 & $100 \mathrm{MPa}$ & $0.001 \mathrm{~J} / \mathrm{m}^{2}$ & $83 \mathrm{GPa}$ & $0.07 \mathrm{GPa}$ \\
\hline
\end{tabular}

Table 1 : Input data for fuel microscopic fragmentation

\section{Conclusion}

A smeared crack model has been proposed to simulate continuously crack initiation and growth with or without an initial flaw. Thanks to a new methodology unstable crack extension can be simulated with a quasi-static nonlinear mechanical approach. This methodology is based on a modified Newton algorithm and uses an adaptive time step strategy coupling to a pseudo dynamic approach to find the post instability solution. Performances and robustness of the method are demonstrated for a full 3D computation to assess nuclear fuel microscopic fragmentation. In this application internal pressure loading is replaced by a fictive thermal loading in order to avoid convergence problem due to the indetermination of the quasi static formulation of a softening material equilibrium under Neumann boundary conditions.

To improve computation time further perspectives can be considered for numerical and computational aspects such as convergence acceleration or parallel computing.

\section{References}

[1] B. Michel, J. Sercombe, G. Thouvenin, R. Chatelet, 3D fuel cracking modelling in pellet cladding mechanical interaction, Engineering Fracture Mechanics 75 (2008) 3581-3598

[2] T. Helfer, Introducing the open-source MFront code generator : application to mechanical behaviours and material knowledgemanagement within the PLEIADES fuel element modelling plateform, Computers and Mathematics with Applications, accepted the 24th June 2015

[3] B. Michel and al., Simulation of the Pellet Cladding Interaction phenomenon with the PLEIADES fuel performance software environment, Nuclear Technology 182 (2), 124-137

[4] B. Michel and al., A new phenomenological criterion for pellet-cladding interaction rupture, Nuclear Engineering and Design 238 (7), 1612-1628

[5] J. Sercombe and al., 1D and 3D analyses of the Zy2 SCIP BWR ramp tests with the fuel codes METEOR and ALCYONE, Nuclear Engineering and Technology, 41(2009) pp. 187-198

[6] C. Esnoul and al., Use of micromechanical approach to investigate transient fuel fragmentation mechanisms, TopFuel 2015 Conference Proceedings - oral presentations - Part II, pp. 476-485

[7] http://www-cast3m.cea.fr/

[8] http://tfel.sourceforge.net/ 